\title{
Long-term efficacy of dabigatran in heparin-induced thrombocytopenia (HIT) after off-pump coronary artery bypass surgery
}

\author{
Łukasz Szyda1, Magdalena Kłosińska1, Natalia Ponińska1, Ewa Mrozowska-Peruga1, \\ Tomasz Kaszczyński², Jarosław D. Kasprzak \\ 1 1st Department of Cardiology, Medical University of Lodz, Bieganski Hospital, Łódź, Poland \\ 2 Department of Cardiac Surgery, Bieganski Hospital, Łódź, Poland
}

Correspondence to: Łukasz Szyda, MD, 1st Department of Cardiology, Medical University of Lodz, Bieganski Hospital, ul. Kniaziewicza 1/5, 91-347 Łódź, Poland, phone: +48422516216 , email: luk.szyda@gmail.com Received: March 3, 2021. Revision accepted: March 23, 2021.

Published online: April 2, 2021. Kardiol Pol. 2021; 79 (4): 479-481 doi:10.33963/KP.15927 Copyright by the Author(s), 2021
Heparin-induced thrombocytopenia (HIT) is a rare immune-mediated disorder related to the use of unfractionated heparin (UFH) and low-molecular-weight heparin (LMWH), associated with an increased risk of vascular thrombosis. Cardiac surgery is one of the major risk factors for HIT. ${ }^{1}$ Parenteral nonheparin anticoagulants-argatroban, danaparoid, bivalirudin, and fondaparinux-are recommended for the treatment of HIT. ${ }^{2}$ The efficacy of dabigatran, a direct oral anticoagulant, is poorly documented in this scenario; in laboratory-confirmed HIT, only a few cases with short-term use were published. ${ }^{3}$

A 71-year-old male, who had undergone off-pump coronary artery bypass surgery (OPCAB) 9 days earlier, with paroxysmal atrial fibrillation in the postoperative period, was transferred to the cardiology department with palpitations and dyspnea lasting for 2 hours. The indication for OPCAB was multivessel coronary artery disease (Supplementary material, Figure S1). On admission to the cardiology department, electrocardiography showed atrial flutter with ventricular rate of $130 \mathrm{bpm}$. Direct current cardioversion was performed with restoration of sinus rhythm.

During OPCAB, 15000 IU of UFH of porcine origin was administered intravenously and after the surgery the patient was anticoagulated with LMWH (nadroparine, $60 \mathrm{mg}$ twice a day subcutaneously). Blood count performed on admission to the cardiology department showed a sudden and significant decrease in platelet

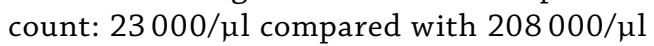

before the cardiac surgery. The unexplained decrease of over $50 \%$ in platelet count 10 days after the cardiac surgery (with intraoperative exposition to UFH) during anticoagulation with nadroparine (FIGURE AA) indicated a high probability of HIT (4Ts score [thrombocytopenia, timing of platelet count fall, thrombosis or other sequelae, other causes for thrombocytopenia] of 6). Nadroparine was immediately stopped and off-label dabigatran therapy was chosen to prevent HIT-related thrombotic events. Considering the early period after the heart surgery, impaired renal function (glomerular filtration rate, $39 \mathrm{ml} / \mathrm{min}$ ) and total bleeding risk in an anticoagulated patient, a reduced dose of dabigatran (110 mg twice a day) was initiated. ${ }^{4}$ The diagnosis of HIT was confirmed by detection of heparin-PF4 antibodies using a latex immunoturbidimetric assay (antibodies level of $15.7 \mathrm{U} / \mathrm{ml}$; reference level, <1.0 U/ml). Deep vein thrombosis was excluded by compression ultrasound (FIGURE 1B-1E). Normalization of platelet count to $234000 / \mu l$ was observed on the fourth day after conversion to dabigatran. Neither thrombotic nor bleeding events occurred during the index hospitalization. Due to a history of paroxysmal atrial fibrillation / atrial flutter $\left(\mathrm{CHA}_{2} \mathrm{DS}_{2}\right.$-VASc score of 6), dabigatran was continued for long-term anticoagulation. After 2 years the patient underwent cryoballoon ablation. On the day of the procedure, the morning dose of dabigatran was skipped, bivalirudin was used intraoperatively, and dabigatran was restored from the evening. During the follow-up period (currently 3 years), 

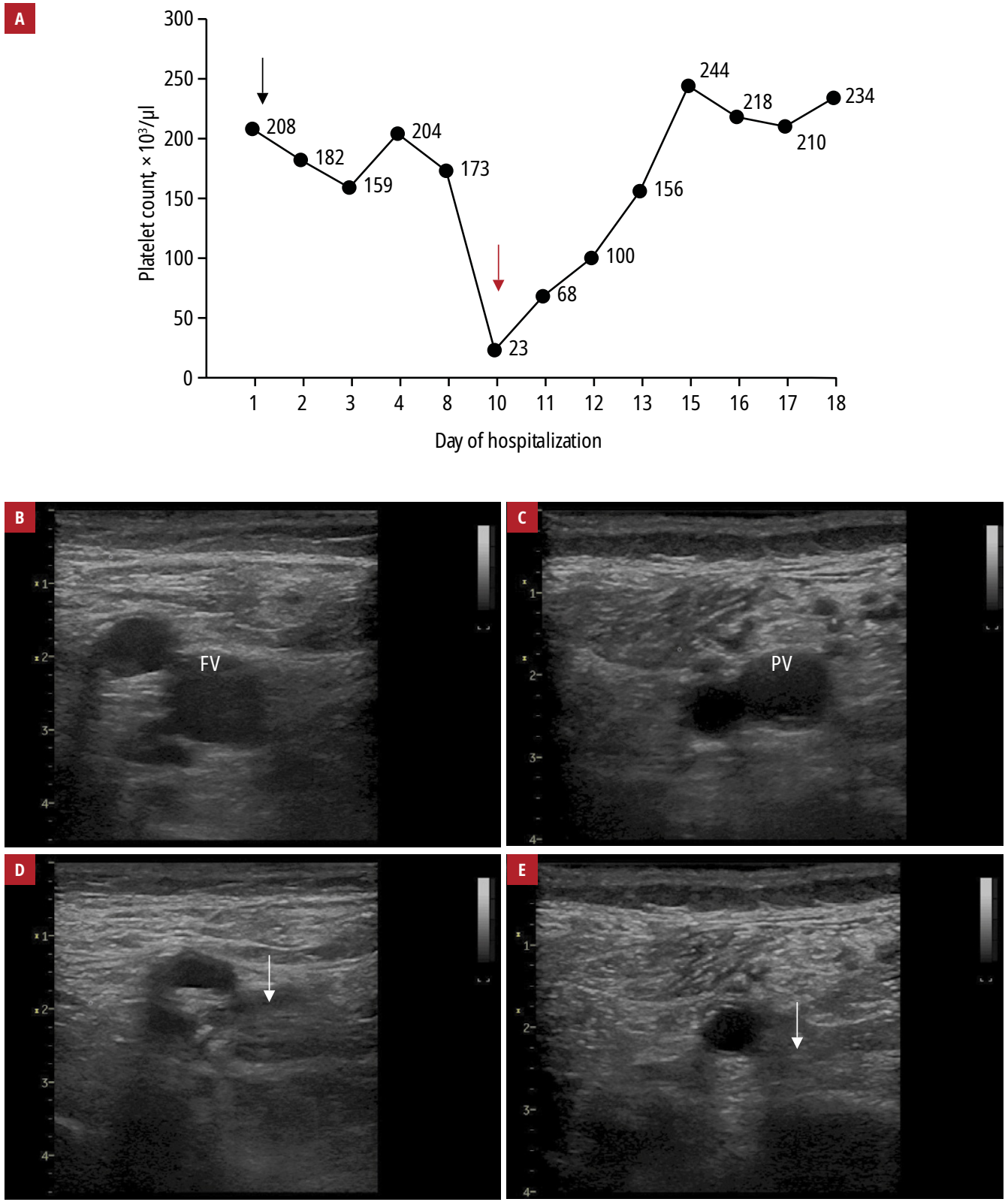

FIGURE 1 A - platelet count during hospitalization; black arrow indicates the day of the off-pump coronary artery bypass surgery (intraoperative use of unfractionated heparin), which was also the beginning of the treatment with nadroparine. In the consecutive days, a sudden, significant decrease in platelet count was observed. Red arrow indicates the switch from nadroparine to dabigatran. B-E - compression ultrasonography of the lower extremity with the right femoral vein (FV) (B) and the right popliteal vein (PV) (C) visible; arrows indicate the compression of the femoral (D) and popliteal (E) veins, which is consistent with the absence of thrombosis.

the patient has been continuously taking dabigatran at a dose of $110 \mathrm{mg}$ twice a day. There was no recurrence of HIT, and no thrombotic or hemorrhagic event was observed.

In the presented case of HIT, dabigatran was used off-label. To our best knowledge, this is the first case of continuous dabigatran use (without conversion to another nonheparin anticoagulant) in laboratory-confirmed HIT with successful long-term follow-up without recurrence. This is also the first case of dabigatran use for the treatment of HIT in a patient early after OPCAB. Our case speaks for long-term efficacy and safety of dabigatran in the clinical scenario of
HIT. Especially during the COVID-19 pandemic, direct oral anticoagulants should be preferred. ${ }^{5}$

\section{SUPPLEMENTARY MATERIAL}

Supplementary material is available at www.mp.pl/kardiologiapolska.

\section{ARTICLE INFORMATION}

\section{CONFLICT OF INTEREST None declared.}

OPEN ACCESS This is an Open Access article distributed under the terms of the Creative Commons Attribution-NonCommercial-NoDerivatives $4.0 \mathrm{In}$ ternational License (CC BY-NC-ND 4.0), allowing third parties to download articles and share them with others, provided the original work is properly cited, not changed in any way, distributed under the same license, and used for noncommercial purposes only. For commercial use, please contact the journal office at kardiologiapolska@ptkardio.pl. 
HOW TO CITE Szyda $Ł$, Kłosińska M, Ponińska N, et al. Long-term efficacy of dabigatran in heparin-induced thrombocytopenia (HIT) after off-pump coronary artery bypass surgery. Kardiol Pol. 2021; 79: 479-481. doi:10.33963/KP.15927

\section{REFERENCES}

1 Greinacher A, Farner B, Kroll H, et al. Clinical features of heparin-induced thrombocytopenia including risk factors for thrombosis. A retrospective analysis of 408 patients. Thromb Haemost. 2005; 94: 132-135.

2 Cuker A, Arepally GM, Chong BH, et al. American Society of Hematology 2018 guidelines for management of venous thromboembolism: heparin-induced thrombocytopenia. Blood Adv. 2018; 2: 3360-3392.

3 Barlow A, Barlow B, Reinaker T, Harris J. Potential role of direct oral anticoagulants in the management of heparin-induced thrombocytopenia. Pharmacotherapy. 2019; 39: 837-853.

4 Undas A, Drabik L, Potpara T. Bleeding in anticoagulated patients with atrial fibrillation: practical considerations. Kardiol Pol. 2020; 78: 105-116.

5 Kosior DA, Undas A, Kopeć G, et al. Guidance for anticoagulation management in venous thromboembolism during the coronavirus disease 2019 pandemic in $\mathrm{Po}$ land: an expert opinion of the Section on Pulmonary Circulation of the Polish Cardiac Society. Kardiol Pol. 2020; 78: 642-646. 